\title{
Robust Scheduling of Residential Distributed Energy Resources Using a Novel Energy Service Decision-Support Tool
}

\author{
M. A. Pedrasa, E. D. Spooner, and I. F. MacGill
}

\begin{abstract}
This paper describes a methodology for making robust day-ahead operational schedules for controllable residential distributed energy resources (DER) using a novel energy service decision support tool. The tool is based on the consumers deriving benefit from energy services and not on electric energy. It maximizes consumer net benefit by scheduling the operation of DER. The robust schedule is derived using a stochastic programming approach formulated for the DER scheduler: the objective function describing the consumer net benefit is maximized over a set of scenarios that model the range of uncertainty. The optimal scenario set is derived using heuristic scenario reduction techniques. Robust operational schedules are formulated for a 'smart' home case study with four controllable DER under stochastic energy service demand, availability of storage DER, and status of dynamic peak pricing. The robust schedule results in a lower expected cost but at the expense of long computation times. The computation period however is not much of a disadvantage because schedules are computed off-line. The consumer can prepare several DER schedules and simply choose the one to implement according to their perception of the coming day. The robust schedules are formulated using an improved version of co-evolutionary particle swarm optimization.
\end{abstract}

Index Terms-Energy services, 'smart' home, distributed energy resources, robust scheduling, scenario trees, scenario reduction, particle swarm optimization.

\section{INTRODUCTION}

The term 'smart' home is increasingly being used to refer to a residential building equipped with embedded intelligence and communication infrastructure that enable automatic response to external and internal stimuli and make pro-active actions with respect to forecast information. This paper describes how an energy service decision-support tool (ES-DST) we previously described in [1] may be used to formulate robust decisions with respect to the operation of residential DER in a 'smart' home. DER are fine-grained equipment and practices that are co-located or near the consumer and can provide some of the functions of the electric utility. The ES-DST is essentially the combination of a novel approach for modeling the demand for, and perceived benefits from, energy services and a DER scheduler that suggests how controllable DER available to the end-user may be operated. In this paper, the ES-DST is used to formulate robust day-ahead operational schedules for various DER under stochastic energy service demand, uncertain availability of some DER, and status of dynamic peak pricing (DPP). The robust schedules are generated by solving the optimization problem associated with the DER scheduler over an optimal set of scenarios that approximates the range of overall uncertainties.

This paper is organized as follows: Section II describes an approach to solving stochastic optimization problems that is applicable to our scheduling problem, Section III describes the ES-DST, Section IV describes the 'smart' home case study and the simulation results, and Section V lists the conclusions.

\section{Stochastic Programming Using Optimal SCENARIO TREES}

\section{A. General Formulation of Problems}

A stochastic programming problem may be described by

$$
\begin{array}{ll}
\min & f_{0}(a, x) \\
\text { s.t. } & f_{i}(a, x) \leq 0, i=1, \ldots, m_{f} \\
& g_{i}(a, x)=0, i=1, \ldots, m_{g} \\
& x \in D_{0}
\end{array}
$$

where $f_{0}$ is the objective function, $f_{i}$ and $g_{i}$ are constraint functions, $x$ is the decision vector that should be within the set $D_{0}$, and $a$ is the vector of stochastic variables [2]. The stochastic optimization problem may be solved by converting it to a substitute deterministic optimization problem. A possible substitute optimization problem for (1) is to discretize the probability distributions of the stochastic variables and to minimize the expected value of $f_{0}$ over a finite set of scenarios that are chosen to model the range of uncertainty [3]. Therefore, the substitute problem is given by

$$
\min \mathrm{E}\left\{f_{0}(x, a)\right\}=\min \sum_{i=1}^{N} \pi\left(a_{i}\right) f_{0}\left(x, a_{i}\right), \sum_{i=1}^{N} \pi\left(a_{i}\right)=1
$$

In these equations, $\pi(a)$ is the probability of the occurrence of the scenario corresponding to a particular realization of $a$.

The range of uncertainty involved in the problem may be approximated by using a large number of scenarios. The computational complexity grows with the number of scenarios therefore their number must be restricted in order to keep the computations within a tractable range [4]. There is also a tradeoff between the accuracy of approximation and the number of scenarios to consider.

\section{B. Generating the Optimal Scenario Set}

The optimal scenario set to which (5) is evaluated may be generated using the following two-step process: 
Step 1: A large number of scenarios is generated by sampling the stochastic variables.

Step 2: The elements of the optimal reduced scenario set (or optimal scenario tree) are chosen from the sample set using the heuristic scenario reduction methods described in [5]: (a) backward scenario reduction and (b) forward scenario selection. These methods are described below.

Several papers have already adopted optimal scenario trees to model the uncertainties in different aspects of power system operation [6]-[8].

1) Backward Scenario Reduction: Scenarios with low probabilities which are very similar to other scenarios with higher probabilities are sequentially removed until the desired set size is reached:

1. Let $S$ be the set of chosen scenarios, initially containing all scenarios. Let $R$ be the set of all deleted scenarios, initially an empty set.

2. Compute the distances $D\left(a_{i}, a_{j}\right)$ between all scenario pairs $\left(a_{i}, a_{j}\right)$ in $S$ :

$$
D\left(a_{i}, a_{j}\right)=\left\|a_{i}-a_{j}\right\|_{2}=\sqrt{\sum_{t=1}^{T}\left(a_{i}^{t}-a_{j}^{t}\right)^{2}}
$$

3. For each scenario $a_{i}$ in $\mathrm{S}$, find the scenario $a_{c}$ closest to it, and compute the utility $U\left(a_{i}\right)$ :

$$
U\left(a_{i}\right)=\pi\left(a_{i}\right) D\left(a_{i}, a_{c}\right)
$$

4. Choose the scenario $a_{i}$ with the smallest utility value

a. Remove $a_{i}$ from $S$ and add it to $R$

b. Update the probability of $a_{c}$ :

$$
\pi\left(a_{c}\right)=\pi\left(a_{c}\right)+\pi\left(a_{i}\right)
$$

5. Repeat steps 3 and 4 until the desired number of scenarios is reached.

2) Forward Scenario Selection: Scenarios with high probabilities which are very similar to a number of low probability scenarios are sequentially added to the optimal scenario set until the desired size is reached:

1. Let $S$ be the set of chosen scenarios, initially an empty set. Let $R$ be the set of all deleted scenarios, initially containing all scenarios.

2. For each scenario $a_{i} \in R$, build a scenario set $P=$ $S \cup\left\{a_{i}\right\}$

a. For each scenario $a_{j} \in R, i \neq j$, compute $D\left(a_{j}, a_{k}\right)$ for all $a_{k} \in P$ using (6)

b. Choose $\min D\left(a_{j}, a_{k}\right)$

c. Compute the utility of scenario $a_{i}, U\left(a_{i}\right)$ :

$$
U\left(a_{i}\right)=\sum_{j=1, a_{j} \notin P}^{N} \pi\left(a_{j}\right) \min D\left(a_{j}, a_{k}\right)
$$

3. Choose the scenario $a_{i}$ with the lowest utility. Add $a_{i}$ to $S$ and remove it from $R$

4. Repeat steps 2 and 3 until the desired number of scenarios is reached

5. Re-compute the probabilities of the chosen scenarios a. For each deleted scenario $a_{i} \in R$, find the chosen scenario $a_{c} \in S$ closest to it

b. Update the probability of $a_{c}$

$$
\pi\left(a_{c}\right)=\pi\left(a_{c}\right)+\pi\left(a_{i}\right)
$$

\section{The Energy Service Decision Support ToOL}

The ES-DST is inspired by the notion that end-users value their energy services and not electricity in its basic form. The energy service model, therefore, is based on putting a monetary benefit to the energy that realizes the service and not to the $\mathrm{kWh}$ of electricity that the end equipment that delivers the service consumes. In this paper, the energy that realizes the service is called the "energy equivalent" of the service being consumed. Examples of "energy equivalents" are the thermal energy that realizes the space heating and hot water services and the mechanical energy output of machines. We first described the ES-DST in [1] and we applied it to formulate operation schedules for a 'smart' home with 3 DER.

The energy service model is composed of a demand model and a benefit model. The demand model is a temporal variation of demand as described by the hourly variation of the "energy equivalents" or any other physical variables identified with the required services (e.g. temperature and liters of hot water). The benefit model is the temporal variation of the benefit derived from the services represented by the hourly value of the monetary benefit derived from each $\mathrm{kWh}$ of the "energy equivalents."

The second component of the ES-DST, or the DER scheduler, formulates the DER operational schedules using the demand and benefit models. The scheduling is essentially an optimization problem that aims to find the DER operation schedule $\boldsymbol{x}$ that maximizes the benefit derived from the energy services less the cost of energy consumption. That is, maximize

$$
\sum_{t=1}^{T} \sum_{i=1}^{S}\left[\lambda_{\mathrm{ES}, i}(t) \cdot U_{\mathrm{ES}, i}(t, \boldsymbol{x})\right]-C
$$

where $\lambda_{E S, i}$ and $U_{E S, i}$ are the perceived benefit and demand for the "energy equivalent" of the $i^{t h}$ service, $C$ is the cost of service provision, $T$ is the number of hours in the simulation period, and $S$ is number of energy services. Many techniques may be used to solve the complex mathematical optimization problem described by (11). In this paper, cooperative particle swarm optimization with stochastic repulsion among particles (CPSO-SR) is used to find $\boldsymbol{x}$. CPSO-SR is an improvement to the canonical formulation of particle swarm optimization that we proposed in an earlier study [9].

Aside from formulating DER operational schedules, we have also used the ES-DST in other applications. In [9], the ES-DST was used to determine the value added by the coordination among the DER. It was used to determine the scenarios where the DER should coordinate their operation and where coordination is not important. In [10], the ES-DST was used to determine the value of making accurate forecasts. This knowledge is important because it identifies the forecast information that are crucial to making effective schedules. 
TABLE I

Mapping of Perceived Benefit of Services to Monetary Values

\begin{tabular}{c|c}
\hline Importance of energy service & Value $(\$ / \mathrm{kWh})$ \\
\hline Must-run & 2.20 \\
High & 1.00 \\
Medium & 0.25 \\
Don't Care & 0.00 \\
Expense & -0.50
\end{tabular}

TABLE II

ELECTRICITY TARIFF

\begin{tabular}{c|c}
\hline Tariff $\left(\lambda_{\mathrm{e}}(t)\right)$ & Rate $(\$ / \mathrm{kWh})$ \\
\hline Time of Use (ToU) & \\
Peak (2 - 8 PM) & 0.3564 \\
Shoulder (7 AM - 2 PM, 8 - 10 PM) & 0.1408 \\
Off-peak (10 PM - 7 AM) & 0.0814 \\
\hline Dynamic peak price (DPP) & \\
High alert (5 - 8 PM) & 2.00 \\
\hline
\end{tabular}

\section{The 'SMART' Home CASE STUdy}

\section{A. Available DER and the Required Energy Services}

The ES-DST is used to formulate robust day-ahead schedules for a 4-DER 'smart' home with stochastic energy service demands, status of DPP, and availability of the plug-in hybrid vehicle (PHEV) as energy storage device. The DER and their operating characteristics are listed below and the first four are scheduled.

1) PHEV. $5.9 \mathrm{kWh}$ capacity, $3.0 \mathrm{~kW}$ maximum charging/discharging rate, $90 \%$ charging/discharging efficiency, may be discharged down to $30 \%$ of capacity, $0.1 \%$ coulomb loss per hour.

2) Space heater. Maximum heating power is $1.8 \mathrm{~kW}$.

3) Storage water heater. Storage capacity is 80 liters and the heating element is rated $1.2 \mathrm{~kW}$.

4) Pool pump. Rated $1.1 \mathrm{~kW}$. Should run at most 6 hours.

5) PV system. Peak output $=2.0 \mathrm{~kW}$.

The DER scheduler determines the hourly charging or discharging rate of the PHEV battery, the hourly heating power of the space heater, the hours when the water heater will be switched on, and the hours when the pool pump is run.

The dollar values assigned to each $\mathrm{kWh}$ of "energy equivalent" of the energy services are listed in Table I. The electricity tariff structure is shown in Table II. Any exported energy is compensated at time-of-use (ToU) rates. These are the ToU rates in Sydney, Australia in 2009, and the DPP rates used by Energy Australia in their strategic pricing study.

The desired indoor temperature when the house is occupied the entire day is shown in Figure 1(a). The actual temperature should be within $1 \mathrm{C}^{\circ}$ from the desired value whenever at least one resident is at home. The residents put a High value to the space heating service. If all residents are away, a Don't Care value is assigned. The "energy equivalent" of the space heating service is the heat content of the indoor air.

The residents may or may not use the PHEV when they leave the house. If they leave the car, the scheduler could use the car battery as a storage DER. The PHEV should be fully charged if they plan to use it. The residents put a High value

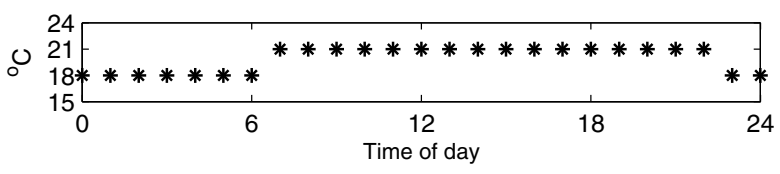

(a)

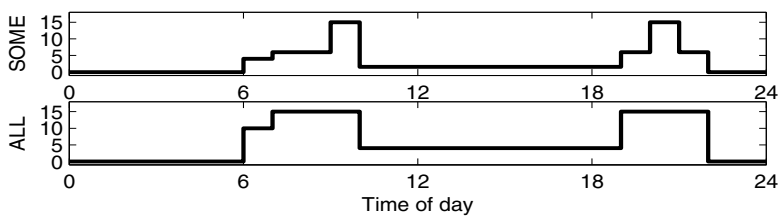

(b)
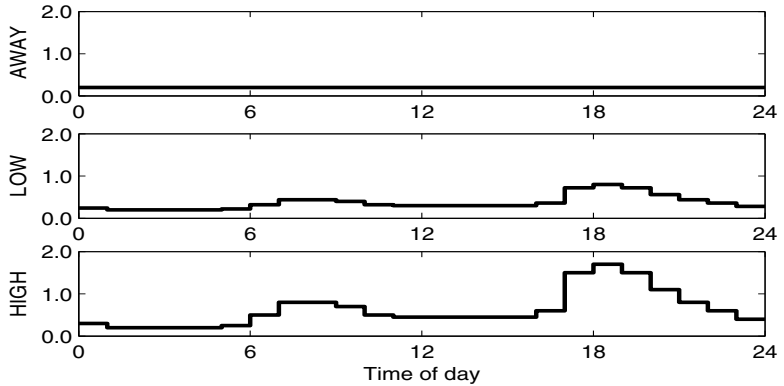

(c)

Fig. 1. Hourly demand for energy services: (a) desired indoor temperature, (b) Hot water demand (liters) at Low and High occupancy states, (c) Must-run energy service demand $(\mathrm{kW})$ for the 3 occupancy states.

to the car charging service, and this benefit applies only to the hour that the residents leave for the first time. On the other hours, a Don't Care value is assigned. The PHEV is fully discharged (30\% of capacity) when the residents return home. The "energy equivalent" of the PHEV charging service is the energy stored in the batteries.

The pool-pump should run at most 6 hours a day anytime from $8 \mathrm{AM}$ to $10 \mathrm{PM}$. The residents assign a Medium value to the pool-pumping service during these hours, and an Expense value outside of this period. An Expense value is assigned to prohibit the pump from running outside the desired period. The energy consumption of the pump is assigned as the "energy equivalent" of the pool pumping service.

All energy services aside from PHEV charging, space and water heating, and pool pumping are lumped together into an aggregate must-run energy service. The demand for hot water and must-run services depends on the level of occupancy of the house. Three hourly occupancy states are assumed: AWAY, SOME and ALL. The residents are all away in the first state, some residents are home in the second state, and all residents are at home in the last state. The must-run and hot water demands are mapped to values corresponding to the occupancy state. The hourly hot water demand when occupancies are SOME and ALL are shown in Figure 1(b). There is no hot water demand when the occupancy is AWAY. The must-run energy service demands are shown in Figure 1(c). The residents put a High value to the hot water service and a Must-run value for the must-run service. The actual energy consumption of the 
must-run service is taken as its "energy equivalent." The heat content of the consumed hot water is the "energy equivalent" of the hot water service.

The relationship between the "energy equivalents" and the actual electricity consumption are described in [1], [9], [10].

\section{B. Models for Stochastic Variables}

The application of the ES-DST to the 'smart' home case study requires forecast information (or predictions) and knowledge of planned consumer actions for it to generate effective DER schedules. However, making accurate predictions especially at the household level is difficult to achieve. Therefore, there is significant value in formulating robust schedules that would produce satisfactory results under forecast uncertainty.

The ES-DST should be able to generate robust schedules under stochastic energy services demands and perceived values. As we have demonstrated in [10], significant additional costs may be incurred if the demand forecast is incorrect.

Aside from magnitude and benefit from the required services, the scheduler should be able to deal with the uncertainty on the following variables: (1) activation of dynamic peak pricing; (2) availability of mobile storage devices (e.g., electric vehicles); (3) solar insolation; and (4) outdoor temperature.

The 'smart' home is under ToU energy tariff with ToU feedin rates and occasional DPP events. In this tariff structure, there is no value in making accurate solar insolation forecasts (as we have demonstrated in [10]), therefore, the variability of solar insolation is not considered. Furthermore, it was assumed that the residents are in possession of accurate outdoor temperature forecasts.

1) Modeling Stochastic Energy Service Demand: The energy service requirements within a household are most dependent to the occupancy of the house. The demand depends on the number of occupants, the times at which the occupants are active, and the times that the occupants are away [11]-[13]. Aside from the presence of occupants, their identities also affect the amount of energy service demand: different occupants have different tendencies in carrying out activities that would require different services. There are several attempts to model these tendencies, one of which is described in [14].

In the absence of an appropriate model that relates occupant presence to energy service demand, the simplest approach is to assume a direct relationship between them. That is, energy service demand is mapped to a particular occupancy level at all hours of the day. This assumption therefore needs a mathematical model for hourly occupancy in dwellings.

Earlier studies have modeled building occupancy as a timeinhomogeneous Markov process in office [15] and domestic buildings [16]. In these studies, the variability of occupancy is modeled as an occupancy transition matrix (OTM). This paper uses a 3-state OTM to model the hourly occupancy in the 'smart' home. The energy service demand in turn depends on the occupancy. This approach to service demand modeling has been utilized before [17].

It is assumed that the OTM is already available and the values are listed in Table III. The entries are the probabilities
TABLE III

OCCUPANCY TRANSITION MATRIX

\begin{tabular}{|c|c|c|c|c|c|c|c|c|c|}
\hline \multirow{2}{*}{ Hour } & \multicolumn{3}{|c|}{ AWAY to } & \multicolumn{3}{c|}{ SOME to } & \multicolumn{3}{c|}{ ALL to } \\
\cline { 2 - 9 } & AWAY & SOME & ALL & AWAY & SOME & ALL & AWAY & SOME & ALL \\
\hline 1 & 0.00 & 0.00 & 1.00 & 0.00 & 0.00 & 1.00 & 0.00 & 0.05 & 0.95 \\
2 & 0.00 & 0.00 & 1.00 & 0.00 & 0.00 & 1.00 & 0.00 & 0.00 & 1.00 \\
3 & 0.00 & 0.00 & 1.00 & 0.00 & 0.00 & 1.00 & 0.00 & 0.00 & 1.00 \\
4 & 0.00 & 0.00 & 1.00 & 0.00 & 0.00 & 1.00 & 0.00 & 0.00 & 1.00 \\
5 & 0.00 & 0.00 & 1.00 & 0.00 & 0.00 & 1.00 & 0.00 & 0.05 & 0.95 \\
6 & 0.00 & 0.00 & 1.00 & 0.00 & 0.95 & 0.05 & 0.05 & 0.05 & 0.90 \\
7 & 0.95 & 0.05 & 0.00 & 0.10 & 0.90 & 0.00 & 0.10 & 0.10 & 0.80 \\
8 & 0.95 & 0.05 & 0.00 & 0.15 & 0.85 & 0.00 & 0.15 & 0.15 & 0.70 \\
9 & 0.95 & 0.05 & 0.00 & 0.30 & 0.70 & 0.00 & 0.30 & 0.30 & 0.40 \\
10 & 0.95 & 0.05 & 0.00 & 0.55 & 0.45 & 0.00 & 0.55 & 0.30 & 0.15 \\
11 & 0.95 & 0.05 & 0.00 & 0.80 & 0.20 & 0.00 & 0.80 & 0.15 & 0.05 \\
12 & 0.95 & 0.05 & 0.00 & 0.95 & 0.05 & 0.00 & 0.95 & 0.05 & 0.00 \\
13 & 1.00 & 0.00 & 0.00 & 1.00 & 0.00 & 0.00 & 1.00 & 0.00 & 0.00 \\
14 & 1.00 & 0.00 & 0.00 & 1.00 & 0.00 & 0.00 & 1.00 & 0.00 & 0.00 \\
15 & 0.95 & 0.05 & 0.00 & 1.00 & 0.00 & 0.00 & 1.00 & 0.00 & 0.00 \\
16 & 0.90 & 0.05 & 0.05 & 0.00 & 0.90 & 0.10 & 1.00 & 0.00 & 0.00 \\
17 & 0.75 & 0.10 & 0.15 & 0.00 & 0.85 & 0.15 & 0.00 & 0.10 & 0.90 \\
18 & 0.50 & 0.25 & 0.25 & 0.00 & 0.75 & 0.25 & 0.00 & 0.05 & 0.95 \\
19 & 0.25 & 0.25 & 0.50 & 0.00 & 0.50 & 0.50 & 0.00 & 0.05 & 0.95 \\
20 & 0.10 & 0.20 & 0.70 & 0.00 & 0.30 & 0.70 & 0.00 & 0.05 & 0.95 \\
21 & 0.05 & 0.15 & 0.80 & 0.00 & 0.20 & 0.80 & 0.00 & 0.05 & 0.95 \\
22 & 0.05 & 0.05 & 0.90 & 0.00 & 0.10 & 0.90 & 0.00 & 0.05 & 0.95 \\
23 & 0.00 & 0.05 & 0.95 & 0.00 & 0.05 & 0.95 & 0.00 & 0.05 & 0.95 \\
24 & 0.00 & 0.00 & 1.00 & 0.00 & 0.05 & 0.95 & 0.00 & 0.05 & 0.95 \\
\hline
\end{tabular}

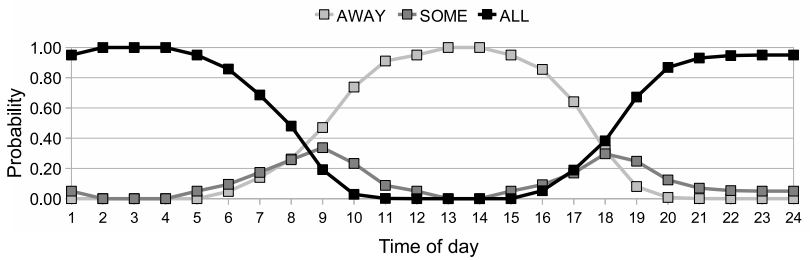

Fig. 2. Probability of the 3 occupancy states at each hour.

that the previous state will change to one of the 3 possible states in the current hour. The entries were chosen such that the house occupancy would follow a typical weekday profile.

The probabilities of the 3 occupancy states every hour may be derived from the OTM. Starting from an ALL initial state (Hour 0), the probability of the 3 states every hour is shown in Figure 2. The figure shows a typical weekday occupancy profile.

2) Forecasting of DPP Events: Electric utilities take into account two factors when deciding to activate DPP events: the projected increase in wholesale prices due to the reduction of generating capacity in the supply side, and the increase of consumption due to weather and social events in the demand side [18]. The supply-side issues are generally not transparent to consumers, therefore, the activation of DPP from their point of view may be only correlated to weather and social factors. To date, we are not aware of any tool that consumers can use to predict the activation of DPP by utilities. Therefore, what consumers can do is to assume with certain confidence that DPP will be announced, and the level of confidence may be based on the weather forecast. In this paper, the consumers express as a probability $\pi_{D}$ that DPP may be active for a 
particular day.

3) Forecasting PHEV Availability as Storage: There are no definite mathematical models that describe the tendency of consumers to use their electric vehicles (or make them available as storage devices) when they leave their homes. In this paper, it is assumed that consumers plan beforehand if they will take the car as they leave. The uncertainty that the PHEV will be available as energy storage is therefore modeled as a probability $\pi_{P}$ that reflects their plan with respect to the car. If they plan to take the car, then the probability of availability is $0 \%$, otherwise, the probability is $100 \%$. If the consumers cannot commit to the availability of the PHEV, then the probability is somewhere between the two extremes, and a $50 \%$ probability means that they are absolutely unsure of their plans.

\section{Formulation of the DER Schedule}

The ES-DST is used to formulate robust DER schedules using the stochastic programming formulation described in Section II. That is, the robust day-ahead schedules are formulated by maximizing (11) using different sets of scenarios that model the variability of occupancy, confidence of DPP prediction and PHEV availability. The scenario sets are derived using both backward scenario reduction and forward scenario selection.

If $A=\left\{a_{1}, \ldots, a_{n}\right\}$ is the set of scenarios that models the range of uncertainty, and each scenario $a_{j}$ has probability $\pi_{j}$, the optimal schedule is derived by

1. Mapping each scenario $a_{j}$ to a corresponding day-ahead energy service demand $d_{j}$. The desired indoor temperature is shown in Figure 1(a) whenever the house is occupied. The hourly hot water and must-run service consumption depends on the hourly occupancy, using Figures 1(b) and (c).

2. Maximizing the objective function

$$
\sum_{j=1}^{20} \pi_{j}\left\{\sum_{t=1}^{T} \sum_{i=1}^{S}\left[\lambda_{\mathrm{ES}, i}(t) \cdot U_{\mathrm{ES}, i}\left(t, \boldsymbol{x}, d_{j}\right)\right]-C\left(\boldsymbol{x}, d_{j}\right)\right\}
$$

The scenario set $A$ has 20 scenarios. A scenario in the set is represented by a 26-element vector and its probability. The first 24 entries $\left(a_{o, 1}, \ldots, a_{o, 24}\right)$ represent the occupancy every hour $(\mathrm{ALL}=1, \mathrm{SOME}=0.5$, AWAY $=0)$, the $25^{\text {th }}$ entry $\left(a_{P}\right)$ is the availability of the PHEV as storage device (available $=1$, not available $=0$ ), and the $26^{\text {th }}$ entry $\left(a_{D}\right)$ is the status of DPP (active $=1$, not active $=0$ ). The scenario set $A$ is generated using a 2-step process:

Step 1: A large number of scenarios are generated by sampling the stochastic variables. A large number of scenarios should be generated in order to capture a large percentage of the entire scenario space, and to achieve a better approximation of uncertainty. A scenario is generated by:

a. Choosing the occupancy sequence by evolving the occupancy states using the OTM. The probability $\pi_{O c c}$ of that sequence is also computed using the OTM.

b. Setting the availability of the PHEV by randomly making the value of $a_{P}$ to 1 or 0 . The probability corresponding $a_{P}$, or $\pi_{P H E V}$, is equal to $\pi_{P}$ if $a_{P}=1$, otherwise it is equal to $1-\pi_{P}$.

c. Setting the DPP status by randomly making the value of $a_{D}$ to 1 or 0 . The probability corresponding to the $a_{D}$, or $\pi_{D P P}$, is equal to $\pi_{D}$ if $a_{D}=1$, otherwise it is equal to $1-\pi_{D}$.

The probability of the generated scenario is therefore equal to

$$
\pi_{i}=\pi_{O c c} \pi_{P H E V} \pi_{D P P} .
$$

After generating $S$ initial scenarios, the scenario probabilities are re-computed such that the total probability is equal to 1 :

$$
\pi_{N}=\sum_{i=1}^{S} \pi_{i} \text { then } \pi_{i}=\frac{\pi_{i}}{\pi_{N}}
$$

Step 2: The elements of the optimal reduced scenario set are chosen from the sample set using the heuristic scenario reduction methods described in Section II-B. Both forward selection and backward reduction methods are implemented and the faster method is chosen. The distance between two scenarios, $D\left(a^{i}, a^{j}\right)$, is computed by

$$
\sqrt{\frac{1}{24}\left\{\sum_{t=1}^{24}\left(a_{o, t}^{i}-a_{o, t}^{j}\right)^{2}\right\}+\left(a_{P}^{i}-a_{P}^{j}\right)^{2}+\left(a_{D}^{i}-a_{D}^{j}\right)^{2}}
$$

The robust DER schedule is labeled as ScenRed. Cooperative PSO with stochastic repulsion (CPSO-SR) is used to determine the schedules. CPSO-SR is briefly described in the appendix. The schedules are formulated using Matlab R2008b, on a $2.0 \mathrm{GHz}$ Intel Pentium Dual Core CPU. The simulation is repeated 10 times and the best schedule is chosen.

\section{Description of Simulation Cases and Baseline Schedules}

Robust schedules are formulated under stochastic occupancy patterns, different confidence levels of DPP prediction, and uncertainty in the plans for making the PHEV available as energy storage. The cases to which robust schedules are formulated are described below.

Case A. The residents are absolutely confident that DPP will not be active for the day, therefore, $\pi_{D}=0.0$. There are two sub-cases:

Case A-1: The residents will take the PHEV when they leave, hence $\pi_{P}=0.0$.

Case A-2: The residents will make the PHEV available as storage, hence $\pi_{P}=1.0$.

Case B. The residents are planning to make the PHEV available as storage, therefore, $\pi_{P}=1.0$. However, they have varying levels of confidence in their DPP predictions. There are three sub-cases:

Case B-1: The residents predict that DPP could be active, but with low probability. Hence $\pi_{D}=0.20$.

Case B-2: The residents are completely unsure on the status of DPP. Hence $\pi_{D}=0.50$.

Case B-3: The residents predict that DPP is most likely to be active. Hence $\pi_{D}=0.80$.

Case C. The residents cannot decide beforehand if they will take the PHEV when they leave, hence $\pi_{P}=0.50$. 
TABLE IV

Comparison Between the Scenario Reduction Methods

\begin{tabular}{|c|c|c|c|c|}
\hline Number of & Sampled & Time to generate & \multicolumn{2}{|c|}{ Time to reduce (mins) } \\
\cline { 4 - 5 } sample & scenario & the initial & Backward & Forward \\
scenarios & space $(\%)$ & scenarios (sec) & reduction & selection \\
\hline 2000 & 73 & 9 & 0.3 & 5.2 \\
3000 & 79 & 20 & 0.5 & 11.8 \\
4000 & 83 & 39 & 1.0 & 21.0 \\
5000 & 86 & 64 & 1.6 & 32.8 \\
\hline
\end{tabular}

Furthermore, they are completely unsure on the status of DPP, hence $\pi_{D}=0.50$.

The simulation cases are chosen to demonstrate the effectiveness of the robust scheduling method against the baseline schedules as the level of uncertainty increases. In Case A, the occupancy is stochastic, and the residents have concrete plans for the PHEV and are very confident with their DPP status prediction. Case B is similar to Case A except that the residents are not perfectly confident on their DPP predictions. Case $\mathrm{C}$ has the highest amount of uncertainty among all cases.

Two baseline schedules are considered for each simulation case. The first baseline schedule, HomeAllDay, assumes the worst case occupancy scenario: the residents are at home the entire day (ALL) so all energy services should be provided. The availability of the PHEV and status of the DPP are the same as that of the simulation case. For example, schedule HomeAllDay for Case A-1 assumes that the PHEV is not available as storage and DPP is not active. The second baseline schedule, ExpectedOcc, is based on the most likely occupancy pattern for the day. Using Fig. 2 as reference, the most likely occupancy is all residents are home (ALL) from 6 PM to $8 \mathrm{AM}$, and all of them are away (AWAY) from 9 AM to 5 PM. Schedule ExpectedOcc is created by solving (12) over 4 scenarios: the most like likely occupancy combined with the 4 possible combinations of DPP and PHEV availability status. The number of scenarios to which ExpectedOcc is created is less than 4 if either $\pi_{P}$ or $\pi_{D}$ is 0 or 1 .

\section{E. Simulation Results: Choosing Between Backward Reduc- tion and Forward Selection Methods}

The backward reduction and forward selection methods are compared with respect to the amount of time it takes them to reduce the initial sample of scenarios. The comparison is shown in Table IV. The first column is the initial number of sampled scenarios. The second column is the percentage of the entire scenario space the initial samples represent. This number is the probability that any randomly generated scenario is part of the initial sample.

The backward reduction method takes a much shorter time to reduce the initial scenario set to 20 scenarios, therefore, it is chosen as the scenario reduction method for ScenRed.

The initial number of scenarios chosen for the succeeding simulations is 3000 . This number is chosen because increasing 3000 to 4000 would only add a small amount to the sampled space (around 4\%) but the reduction time would double (from 0.5 to 1 minute). Furthermore, the increase from 2000 scenarios to 3000 added around $6 \%$ to the sampled space but only 0.2 minutes to the simulation time.

\section{F. Simulation Results: Formulation of Robust DER Schedules}

The robust and baseline schedules are compared to each other by evaluating them against large sets of scenarios that are realizations of the stochastic variables. In this paper, these sets are called evaluation sets, and each evaluation set contains 10,000 unique scenarios. The sum of the probabilities of all 10,000 scenarios is around $92 \%$. The evaluation sets are generated by evolving the occupancy states for the entire day using the occupancy transition matrix and combining them with particular PHEV availability and DPP states. Therefore, there are four evaluation sets:

ES-1: PHEV not available and no DPP $\left(a_{P}=0, a_{D}=0\right)$

ES-2: PHEV not available and with DPP $\left(a_{P}=0, a_{D}=1\right)$

ES-3: PHEV available and no DPP $\left(a_{P}=1, a_{D}=0\right)$

ES-4: PHEV available and with DPP $\left(a_{P}=1, a_{D}=1\right)$

The comparisons are shown in Table V. The tables show the expected costs when the schedules are evaluated across the relevant evaluation sets and the time to generate the schedules. When a schedule is evaluated over a scenario, the cost is equal to the sum of the cost of electricity, the cost of undelivered services, and the cost of battery discharge (computed using the cost of a battery over its useful life, based on the maximum number of discharge cycles), less the value of the energy left in the storage devices (PHEV battery and hot water tank). This formula is adopted to reflect the inconvenience caused by undelivered services and the cost of energy remaining in the storage devices.

Table $\mathrm{V}$ shows the expected costs for Case A and the evaluation sets used to compare the schedules. In Case B, if the tendency for DPP is unknown (i.e., it can go either way), then the expected costs for ES-3 and ES-4 may be averaged to determine the expected costs. All schedules are effectively evaluated across 20,000 scenarios belonging to both scenario sets. Using the same argument in Case C, the expected costs for the four evaluation sets may be averaged if the tendency for DPP and the availability of the PHEV cannot be determined. The schedules are therefore evaluated across the 40,000 scenarios of the 4 evaluation sets.

The results show that ScenRed is the best schedule based on the expected cost. The large number of scenarios used to maximize the net benefit is able to capture a wider range of stochasticity, as suggested by the optimal scenario set derived for Case A-1 in Table VI. The optimal scenario set includes scenarios with different departure and arrival times, and depicts occupancy transitions between ALL and SOME, and SOME and AWAY on some scenarios. However, ScenRed takes the longest to generate because it is optimized over 20 different occupancy, PHEV availability, and DPP scenarios. On the other hand, HomeAllDay is optimized over 1 scenario while ExpectedOcc is optimized over at most 4 scenarios. The results also show that in some cases, it is better to assume the worst occupancy scenario (HomeAllDay), than the most likely scenario (ExpectedOcc). 
TABLE V

Simulation Results: Expected Costs and Simulation Times

Case A:

\begin{tabular}{|c|c|c|c|c|}
\hline Case & HomeAllDay & ExpectedOcc & ScenRed & Eval. Set \\
\hline A-1 & $\$ 6.65(4.3$ mins $)$ & $\$ 4.66(4.0 \mathrm{mins})$ & $\$ 4.00(60.3 \mathrm{mins})$ & ES-1 \\
A-2 & $\$ 3.04(4.2 \mathrm{mins})$ & $\$ 3.41(4.0 \mathrm{mins})$ & $\$ 3.02(62.4 \mathrm{mins})$ & ES-3 \\
\hline
\end{tabular}

Case B:

\begin{tabular}{|c|c|c|c|}
\hline Evaluation set: & ES-3 & ES-4 & $50-50$ \\
\hline HomeAllDay(4.1 mins) & $\$ 3.60$ & $\$ 3.64$ & $\$ 3.62$ \\
\hline \multicolumn{4}{|c|}{ Case B-1: DPP prediction $=20 \%$} \\
\hline ExpectedOcc $(6.8 \mathrm{mins})$ & $\$ 3.69$ & $\$ 3.76$ & $\$ 3.72$ \\
\hline ScenRed (66.7 mins) & $\$ 3.06$ & $\$ 3.51$ & $\$ 3.28$ \\
\hline \multicolumn{4}{|c|}{ Case B-2: DPP prediction $=50 \%$} \\
\hline ExpectedOcc (6.9 mins) & $\$ 3.67$ & $\$ 3.83$ & $\$ 3.75$ \\
\hline ScenRed (60.2 mins) & $\$ 3.12$ & $\$ 3.25$ & $\$ 3.18$ \\
\hline \multicolumn{4}{|c|}{ Case B-3: DPP prediction $=80 \%$} \\
\hline ExpectedOcc (7.1 mins) & $\$ 3.68$ & $\$ 3.74$ & $\$ 3.71$ \\
\hline ScenRed (63.7 mins) & $\$ 3.16$ & $\$ 3.24$ & $\$ 3.20$ \\
\hline
\end{tabular}

Case C:

\begin{tabular}{|l|l|l|l|l|l|}
\hline \multicolumn{1}{|c|}{ Evaluation set: } & ES-1 & ES-2 & ES-3 & ES-4 & 25-all \\
\hline HomeAllDay (4.1 mins) & $\$ 4.79$ & $\$ 7.94$ & $\$ 3.60$ & $\$ 3.64$ & $\$ 4.99$ \\
ExpectedOcc (11.4 mins) & $\$ 4.76$ & $\$ 9.20$ & $\$ 3.40$ & $\$ 3.51$ & $\$ 5.28$ \\
ScenRed (58.5 mins) & $\$ 4.19$ & $\$ 7.94$ & $\$ 3.16$ & $\$ 3.22$ & $\$ 4.63$ \\
\hline
\end{tabular}

The time to generate the schedules are enclosed in parenthesis.

TABLE VI

Optimal Reduced SCEnARIo Set For CASE A-1

\begin{tabular}{|c|c|c|c|c|}
\hline Scenario ID & $\begin{array}{c}\text { Probability } \\
(\%)\end{array}$ & Hourly Occupancy: & $a_{P}$ & $a_{D}$ \\
\hline$a_{r 1}$ & 10.2 & & 1 & 0 \\
\hline$a_{r 2}$ & 7.4 & & 1 & 0 \\
\hline$a_{r 3}$ & 7.2 & & 1 & 0 \\
\hline$a_{r 4}$ & 6.8 & & 1 & 0 \\
\hline$a_{r 5}$ & 6.6 & & 1 & 0 \\
\hline$a_{r 6}$ & 6.1 & & 1 & 0 \\
\hline$a_{r 7}$ & 5.8 & & 1 & 0 \\
\hline$a_{r 8}$ & 5.2 & & 1 & 0 \\
\hline$a_{r 9}$ & 5.1 & & 1 & 0 \\
\hline$a_{r 10}$ & 4.7 & & 1 & 0 \\
\hline$a_{r 11}$ & 4.7 & & 1 & 0 \\
\hline$a_{r 12}$ & 4.3 & & 1 & 0 \\
\hline$a_{r 13}$ & 4.3 & & 1 & 0 \\
\hline$a_{r 14}$ & 3.6 & & 1 & 0 \\
\hline$a_{r 15}$ & 3.5 & & 1 & 0 \\
\hline$a_{r 16}$ & 3.2 & & 1 & 0 \\
\hline$a_{r 17}$ & 3.2 & & 1 & 0 \\
\hline$a_{r 18}$ & 3.1 & & 1 & 0 \\
\hline$a_{r 19}$ & 2.9 & & 1 & 0 \\
\hline$a_{r 20}$ & 2.3 & & 1 & 0 \\
\hline
\end{tabular}

* White = AWAY; light gray $=$ SOME; dark gray = ALL .

The lower expected cost for ExpectedOcc may be explained using the DER operational schedules for Case $\mathrm{C}$ shown in Fig. 3. The expected cost is reduced by (a) charging the PHEV battery toward the end of the off-peak period (to minimize leakage) and discharging the stored energy during the DPP period to supply energy that is otherwise bought from the grid; (b) preheating the house when the residents leave to minimize the heater consumption during the peak period; (c) maintaining the indoor temperature within the comfortable range during the

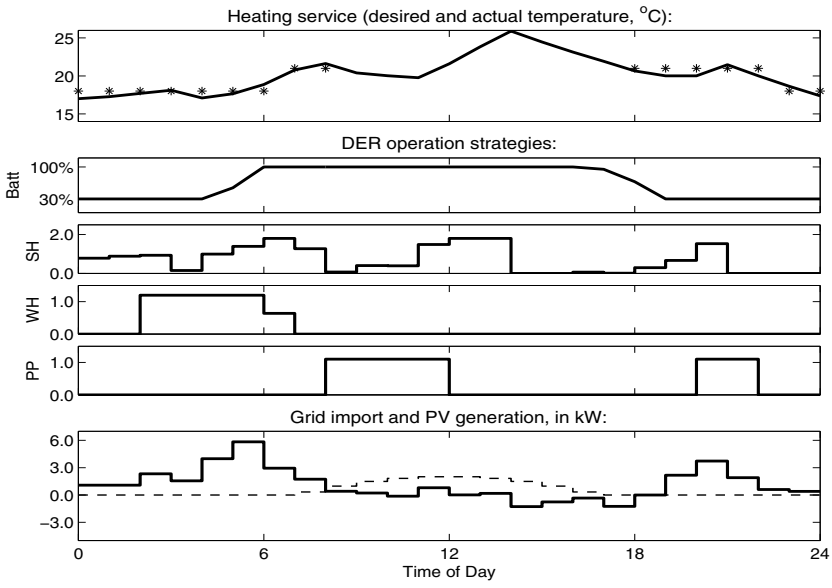

Fig. 3. ExpectedOcc DER operational schedules for Case C. Shown are the desired and actual indoor temperatures, DER schedules, and total grid import and PV output. The DER operation schedules are described by the stored energy in the PHEV battery (Batt, \% of full capacity), space heater power $(\mathrm{SH}$, in $\mathrm{kW})$, water heater power $(\mathrm{WH}$, in $\mathrm{kW})$, and pool pump power ( $\mathrm{PP}$, in $\mathrm{kW}$ ).

morning hours in anticipation of the variable departure times; and (d) heating an adequate amount of water in anticipation of variable occupancy periods. The higher expected cost in the other schedules is due to (a) the failure to utilize the PHEV as energy storage (e.g., in Case A-1); (b) the inability to preheat the house by the HomeAllDay schedule; (c) the inability to provide all required energy services by the ExpectedOcc schedule (e.g., room temperature beyond comfortable range when the residents leave late and inadequate hot water during high occupancy days).

\section{CONCLUSION}

This paper demonstrates that robust day-ahead DER operation schedules may be generated using the ES-DST by formulating a stochastic programming problem for the DER scheduler. The schedule is derived by maximizing the net benefit function over a set of scenarios that model uncertain energy service demand, DPP status, and availability of the PHEV as energy storage device.

The simulation results for the 'smart' home case study show that the robust schedule derived using stochastic programming results in lower expected cost than baseline schedules that assume the worst case scenario and the most likely occupancy pattern. The ScenRed schedule, however, takes the longest time (around 1 hour) to generate because the objective function is maximized over 20 different scenarios. On the other hand, it only takes 4 minutes to generate the HomeAllDay schedule, and 4 to 11 minutes to generate the ExpectedOcc schedule depending on the level of uncertainty. The long computation time is not much of a disadvantage because the schedules are computed off-line. In fact, in a practical application the residents can prepare several DER schedules (each optimized for a probability of DPP activation or PHEV availability [and even occupancy] uncertainty), and simply choose the one to implement according to their perception of the coming day. To 
illustrate, if the consumers are definite that they will make the PHEV available as storage, but are uncertain of the probability of DPP, they can simply choose which among the precomputed schedules to implement: they can choose among the schedules for Cases B-1, B-2 or B-3, or even choose the schedule for Case A-2 if they think that DPP probability is closer to $0 \%$ than $20 \%$. Furthermore, if they are not certain on the availability of the PHEV, they can implement the schedule for Case C. They can even optimize schedules for different tendencies with respect to PHEV availability, similar to the different probabilities for the DPP. All of these schedules may be prepared days, or even weeks ahead of time.

The scheduling method and results presented in this paper are all preliminary. The objective is only to demonstrate that the ES-DST maybe utilized to create robust day-ahead DER schedules. Further studies may be undertaken, some of which are: (a) analysis for other tariff structures (e.g. peak demand charges, net feed-in tariff, and real-time pricing); (b) incorporation of more stochastic variables (e.g. weather); (c) mathematical formulation using another stochastic programming approach (e.g. chance-constrained formulation); (d) improvement of the selection method for the reduced scenario set; and (e) adoption of other algorithms to optimize the objective function over the the optimal scenario sets. While practical implementation may be some way off, the work highlights the potential value of focusing on robust automated scheduling of DER in future 'smart' homes where there will be considerable uncertainties to manage.

\section{APPENDIX \\ CoOperative PSO With Stochastic Repulsion AMONG PARTICLES}

Particle swarm optimization (PSO) is a search technique that belongs to a class of heuristic optimization algorithms that mimic how a group of simple particles could achieve complex collective behaviors. In PSO, a particle represents a candidate solution and the particles are made to wander around the solution space. The movement of the particles are guided by the following speed and position equations:

$$
\begin{gathered}
v_{i}{ }^{t+1}=\omega v_{i}{ }^{t}+c_{1} r()\left(x_{P b, i}{ }^{t}-x_{i}{ }^{t}\right)+c_{2} r()\left(x_{G b, i}{ }^{t}-x_{i}{ }^{t}\right) \\
x_{i}{ }^{t+1}=x_{i}{ }^{t}+v_{i}{ }^{t+1}
\end{gathered}
$$

CPSO-SR is an improvement of the canonical formulation of PSO that we proposed in [9]. In cooperative PSO, the variables are optimized using a divide-and-conquer approach: the variables to be optimized are divided into several groups, and a swarm is used to optimize each group [19]. We introduced repulsion among the particles to discourage early convergence and to allow the particles to cover a larger region of the solution space. Repulsion is achieved by allowing a coordinate to move away from the global and personal best positions, and this behavior is conditional to a probability which in turn depends on the iteration number. When a coordinate moves away from the other particles, the speed is computed by $v_{i}{ }^{t+1}=-\omega v_{i}{ }^{t}-c_{1} r()\left(x_{P b, i}{ }^{t}-x_{i}{ }^{t}\right)-c_{2} r()\left(x_{G b, i}{ }^{t}-x_{i}{ }^{t}\right)$.

The simulations showed that CPSO-SR can achieve better results than canonical cooperative PSO.

\section{ACKNOWLEDGMENT}

Michael Pedrasa acknowledges the scholarship granted by the University of the Philippines through the DOST-ERDT and UP DSF, and the support from the Australian Solar Institute.

\section{REFERENCES}

[1] M. Pedrasa, E. Spooner, and I. MacGill, "Improved energy services provision through the intelligent control of distributed energy resources," in IEEE Bucharest PowerTech, Jun/Jul 2009.

[2] K. Marti, Stochastic Optimization Methods, 2nd ed. Berlin, Germany: Springer, 2008.

[3] M. Kaut and S. W. Wallace, "Evaluation of scenario-generation methods for stochastic programming," Available at http://edoc.huberlin.de/series/speps/2003-14/PDF/14.pdf, 2003.

[4] H. Heitsch and W. Romisch, "Generation of multivariate scenario trees to model stochasticity in power management," in 2005 IEEE Russia PowerTech Conf., June 2005.

[5] J. Dupacovà, N. Grôwe-Kuska, and W. Rőmisch, "Scenario reduction in stochastic programming," Math. Programming, vol. 95, no. 3, pp. 493-511, 2003

[6] L. Wu, M. Shahidehpour, and T. Li, "Stochastic security-constrained unit commitment," IEEE Trans. on Power Syst., vol. 22, no. 2, pp. 800-811, May 2007.

[7] V. Pappala, I. Erlich, K. Rohrig, and J. Dobschinski, "A stochastic model for the optimal operation of a wind-thermal power system," IEEE Trans. on Power Sys., vol. 24, no. 2, pp. 940-950, May 2009.

[8] J. M. Latorre, S. Cerisola, and A. Ramos, "Clustering algorithms for scenario tree generation: Application to natural hydro inflows," European J. of Operational Research, vol. 181, no. 3, pp. 1339-1353, 2007.

[9] M. Pedrasa, E. Spooner, and I. MacGill, "Coordinated scheduling of residential distributed energy resources to optimize 'smart' home energy services," IEEE Tran. on Smart Grid, vol. 1, no. 2, pp. 134-143.

[10] — "The value of accurate forecasts and a probabilistic method for robust scheduling of residential distributed energy resources," in 11th Int. Conf. on Probabilistic Methods Applied to Power Syst., June 2010, pp. 587-592.

[11] S. Firth, K. Lomas, A. Wright, and R. Wall, "Identifying trends in the use of domestic appliances from household electricity consumption measurements," Energy and Buildings, vol. 40, no. 5, pp. 926-936, 2008.

[12] Y. G. Yohanis, J. D. Mondol, A. Wright, and B. Norton, "Real-life energy use in the UK: How occupancy and dwelling characteristics affect domestic electricity use," Energy and Buildings, vol. 40, no. 6, pp. 10531059, 2008.

[13] R. Yao and K. Steemers, "A method of formulating energy load profile for domestic buildings in the UK," Energy and Buildings, vol. 37, no. 6 , pp. 663-671, 2005.

[14] A. Capasso, W. Grattieri, R. Lamedica, and A. Prudenzi, "A bottom-up approach to residential load modeling," IEEE Trans. on Power Syst. vol. 9, no. 2, pp. 957-964, May 1994.

[15] J. Page, D. Robinson, N. Morel, and J.-L. Scartezzini, "A generalised stochastic model for the simulation of occupant presence," Energy and Buildings, vol. 40, no. 2, pp. 83-98, 2008.

[16] I. Richardson, M. Thomson, and D. Infield, "A high-resolution domestic building occupancy model for energy demand simulations," Energy and Buildings, vol. 40, no. 8, pp. 1560-1566, 2008.

[17] J. Widen, A. M. Nilsson, and E. Wackelgard, "A combined Markov-chain and bottom-up approach to modelling of domestic lighting demand," Energy and Buildings, vol. 41, no. 10, pp. 1001-1012, 2009.

[18] S. Braithwait, "Behavior modification," IEEE Power and Energy Mag., vol. 8, no. 3, pp. 36-45, May-Jun 2010

[19] F. van den Bergh and A. Engelbrecht, "A cooperative approach to particle swarm optimization," IEEE Tran. on Evol. Computat., vol. 8, no. 3, pp. 225-239, June 2004. 